\title{
Extracorporeal Membrane Oxygenation in Coronavirus induced Severe Acute Respiratory Distress Syndrome
}

\author{
Sarbjot Grewal ${ }^{1 *}$, Kamalmeet Kaur ${ }^{2}$, Ali S Rad ${ }^{3}$, Amritpal S Nat $^{3}$ and Pankaj Bharati ${ }^{3}$ \\ ${ }^{1}$ Internal Medicine residency, Saint Agnes Medical Center, USA \\ ${ }^{2}$ Internal Medicine residency, University of California, USA \\ ${ }^{3}$ Pulmonary and Critical Care, Saint Agnes Medical Center, USA \\ *Corresponding author: Sarbjot Grewal, Internal Medicine residency, Saint Agnes Medical Center, 1303 E. Herndon Avenue, Fres- \\ no, CA. 93725, USA.
}

To Cite This Article: Sarbjot Grewal, Kamalmeet Kaur, Ali S Rad, Amritpal S Nat, Pankaj Bharati, Extracorporeal Membrane Oxygenation in Coronavirus induced Severe Acute Respiratory Distress Syndrome. Am J Biomed Sci \& Res. 2021 - 12(3). AJBSR.MS.ID.001745.

DOI: 10.34297/AJBSR.2021.12.001745.

Received: 䟧 March 10, 2021; Published: 眥 March 22, 2021

\begin{abstract}
Rationale: VV-ECMO is primarily employed in refractory hypoxemic respiratory failure, but limited data is available regarding its use in SARSCoV-2 induced ARDS.
\end{abstract}

Objectives: To evaluate the role of ECMO in SARS-CoV-2 positive patients.

Methods: We present a case report of a young female with SARS-CoV-2 induced ARDS, who received ECMO.

Main results: ECMO may be employed in younger, healthy patients with limited co-morbidities as in our patient.

Conclusions: We present a successful ECMO application in a COVID -19 positive patient with severe ARDS

\section{Introduction}

The ongoing coronavirus pandemic presents an unprecedented challenge to the healthcare system. Originating in Wuhan, China, severe acute respiratory syndrome coronavirus 2 (SARS-CoV-2) has spread rapidly and extensively and has overwhelmed hospital systems around the world. COVID-19 associated respiratory failure requiring intubation and mechanical ventilation is treated by low lung volume ventilation strategies per ARDS-NET protocol [1]. When patients develop refractory hypoxemia despite standard care and proning, the next available intervention is Extracorporeal membrane oxygenation (ECMO). However, mortality benefit from employing ECMO in COVID 19 is still unclear. Here we describe a case of a young, obese SARS- CoV-2 positive patient who benefited from the use of ECMO.

\section{Case presentation}

A 35-year-old obese female with BMI of 35, non-smoker with no known co-morbidities presented to her PCP's office with symptoms of cough, shortness of breath. The patient was treated for asthma exacerbation but due to worsening symptoms, she presented to the Emergency Room. Upon admission, the patient was found to be febrile, tachycardia, tachypneic with oxygen saturation at $94 \%$ on room air. Auscultation of chest revealed coarse breath sounds bilaterally. As shown in Figure 1, Chest X ray shows bibasilar opacities. On admission, patient was started on treatment of presumed community acquired pneumonia, patient was also tested for SARS-Cov-2 with RT-PCR via a nasopharyngeal swab on 
admission. Patient received IV antibiotics for 3 days, on Day 4 of hospital stay, RT-PCR result confirmed SARS-Cov-2 infection.

Patient's oxygen saturation dropped below $90 \%$ on Day 4 of stay, requiring initially $2 \mathrm{~L}$ oxygen via nasal cannula, subsequently increased to $6 \mathrm{~L} \mathrm{NC}$ later the same day. Patient continued to be hypoxemic and was started on heated high flow with FiO2 at 0.65 and $35 \mathrm{~L} / \mathrm{min}$ along with awake proning measures which resulted in minimal improvement of oxygenation. On Day 8 patient underwent emergent intubation and mechanical ventilation and required vasopressor support. Interleukin- 6 and CRP checks that day were elevated at 331.3 and $226 \mathrm{mg} / \mathrm{L}$ respectively. Due to her rapidly deteriorating condition, the patient qualified for compassionate use of an IL-6 inhibitor Tocilizumab on day 9 but showed no improvement. Even after employing mechanical ventilation, the patient was desaturating, thus a paralytic was administered which resulted in temporary improvement in oxygenation with saturation improving to $95 \%$. After 3 days of continued ventilator support and proning, worsening oxygenation was noted, her saturations were averaging at $85 \%$, thus the decision was made to institute Venovenous ECMO on day 13. The patient was cannulated in our hospital and transferred to a close ECMO center of excellence facility. She was on ECMO for 10 days and was successfully de-cannulated on
Day 23 and successfully extubated on Day 28.

\section{Discussion}

Veno-venous ECMO is primarily used in patients with refractory hypoxemic respiratory failure. In the recent decades, few studies attempted to evaluate the consideration of ECMO in the critically ill. These events included the H1N1 pandemic, CESAR (Efficacy and Economic Assessment of Conventional Ventilatory Support versus Extracorporeal Membrane Oxygenation for Severe Adult Respiratory Failure).

CESAR trial in 2006 compared 90 patients with and without ECMO, showed a survival rate with a 6 month follow up, was $63 \%$ and $47 \%$ respectively [2]. In a study during the H1N1 pandemic in 2008 comparing outcomes of patients receiving ECMO supported the hypothesis that subjects with severe co-morbidities and results of multi-organ failure have a higher mortality rate [3,4]. After the CESAR trial, the data shows an increasing trend in the development of ECMO centers and the use of ECMO in critically ill patients. Studies done during the MERS epidemic in 2012 showed some mortality benefit associated with ECMO as well [5-7]. However, the EOLIA in 2018 trial did not show a 60-day mortality benefit for ECMO compared to standard of care which included proning and low tidal volume lung ventilation strategies [8] (Figure 2).

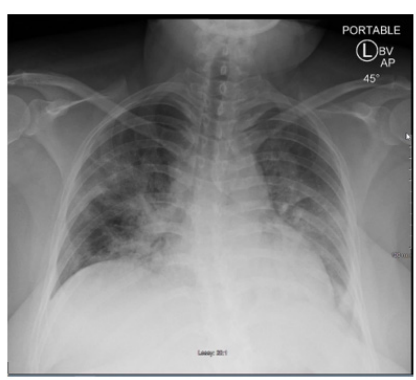

Figure 1

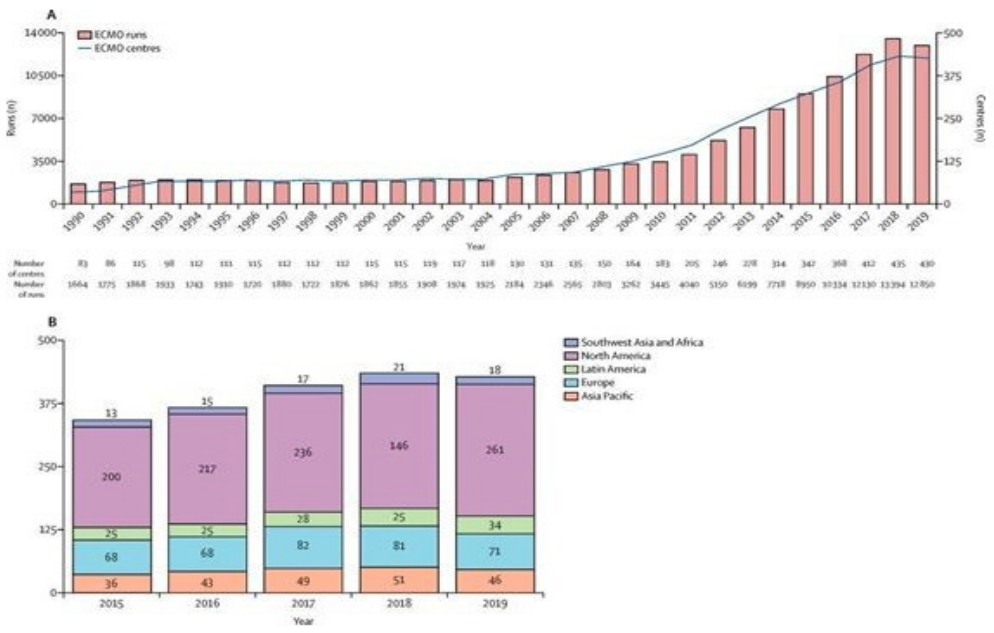

Figure 2 
Patients who develop ARDS with refractory hypoxemic respiratory failure may qualify for ECMO, but whether or not ECMO can confer a mortality benefit in the presence of significant comorbidities is unclear. Given the great expense of ECMO and the potential for nosocomial infections or intracranial hemorrhage, its use in certain patient populations, especially elderly patients with multiple comorbidities, should be conservative if employed at all as shown in a 2013 systematic review analyzing ECMO use in H1N1 patients [4]. It is imperative to emphasize the need for trained staff, appropriate infrastructure, and proper disposable equipment in order to successfully employ ECMO. Advanced preparations with an ECMO center helped safely and efficiently transfer this patient to a higher level of care. This pandemic calls for a collaborative effort among all physicians for best management of patients.

\section{Conclusion}

We report a case of ECMO deployment in a young female with SARS-CoV-2 induced refractory hypoxic respiratory failure who failed standard of care treatment with proning. Aside from ethnicity, age, and comorbid factors, further information such as the duration of infection or severity of viral load before treatment can be explored as more data is collected to help characterize patients who would benefit from ECMO.

Our case exemplifies that carefully selected patients with refractory hypoxemia from COVID-19 may benefit from ECMO at specialized centers. It is vital to emphasize that further studies are required to better define the role of ECMO in the current fight against the novel Coronavirus.

\section{References}

1. Mohammed S Alshahrani, Anees Sindi, Fayez Alshamsi, Awad Al-Omari, Mohamed El Tahan, et al. (2018) Extracorporeal Membrane Oxygenation for Severe Middle East Respiratory Syndrome Coronavirus. Annals of Intensive Care 8(1): 3.

1. Henry, Giuseppe Lippi (2020) Poor Survival with Extracorporeal Membrane Oxygenation in Acute Respiratory Distress Syndrome (ARDS) due to Coronavirus Disease 2019 (COVID-19): Pooled Analysis of Early Reports. Journal of Critical Care 58: 27-28.

2. Zangrillo, Alberto, Giuseppe Biondi-Zoccai, Giovanni Landoni, Giacomo Frati, Nicolò Patroniti, et al. (2013) Extracorporeal Membrane Oxygenation (ECMO) in Patients with H1N1 Influenza Infection: a Systematic Review and Meta-Analysis Including 8 Studies and 266 Patients Receiving ECMO. Critical Care 17(1): R30.

3. Jahangirifard, Alireza, Zargham Hossein Ahmadi, Majid Golestani Eraghi, Payam Tabarsi, Majid Marjani, et al. (2016) H1N1 Influenza Patient Saved by Extracorporeal Membrane Oxygenation: First Report from Iran. The Journal of Tehran Heart Center 11(3): 153-156.

4. Brodie Daniel (2018) The Evolution of Extracorporeal Membrane Oxygenation for Adult Respiratory Failure. Annals of the American Thoracic Society 15(Suppl 1): S57-S60.

5. Peek GJ, Mugford M, Tiruvoipati R, Wilson A, Allen E, et al. (2009) Efficacy and economic assessment of conventional ventilatory support versus extracorporeal membrane oxygenation for severe adult respiratory failure (CESAR): a multicentre randomised controlled trial. Lancet 374(9698): 1351-1363.

6. Mishra, Jan L Svennevig, Jan F Bugge, Sølvi Andresen, Agnete Mathisen, et al. (2010) Cost of Extracorporeal Membrane Oxygenation: Evidence from the Rikshospitalet University Hospital, Oslo, Norway. Eur J Cardiothorac Surg 7(2): 339-342.

7. Combes Alain, David Hajage, Gilles Capellier, Alexandre Demoule, Sylvain Lavoué, et al. (2018) Extracorporeal Membrane Oxygenation for Severe Acute Respiratory Distress Syndrome: NEJM. New England Journal of Medicine 378(21): 1965-1975. 